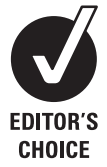

Correspondence to Dr Jeremy S Lewis, Therapy Department, Chelsea and Westminster NHS Healthcare, 369 Fulham Road, London SW10 9NH, UK; jeremy. lewis@chelwest.nhs.uk

Accepted 31 March 2009

\title{
Rotator cuff tendinopathy: a model for the continuum of pathology and related management
}

\author{
Jeremy S Lewis
}

\begin{abstract}
Background Pathology of the soft tissues of the shoulder including the musculotendinous rotator cuff and subacromial bursa are extremely common and are a principal cause of pain and suffering. Competing theories have been proposed to explain the pathoaetiology of rotator cuff pathology at specific stages and presentations of the condition. This review proposes a model to describe the continuum of the rotator cuff pathology from asymptomatic tendon through full thickness rotator cuff tears.

Conclusions The pathoaetiology of rotator cuff failure is multifactorial and results from a combination of intrinsic, extrinsic and environmental factors. Recently a new and generic model detailing the continuum of tendon pathology has been proposed. This model is relevant for the rotator cuff and provides a framework to stage the continuity of rotator cuff pathology. Furthermore, it provides a structure to identify the substantial deficiencies in our knowledge base and areas where research would improve our understanding of the pathological and repair process, together with assessment and management. The strength of this model adapted for the rotator cuff tendons and subacromial bursa will be tested in its ability to incorporate and adapt to emerging research.
\end{abstract}

\section{INTRODUCTION}

Certain sports place substantial demands on the shoulder. Elite swimmers execute approximately 2 million strokes per year, ${ }^{2}$ and professional baseball pitchers generate ball speeds of up to $165 \mathrm{~km} / \mathrm{h}$ with associated peak internal-rotation velocities up to $6940 \% \mathrm{~s}^{3}{ }^{4}$ These data elucidate why pathologies of the musculotendinous rotator cuff and subacromial bursa (SAB) are considered principal causes of shoulder pain. Various theories have been proposed to explain the pathogenesis of rotator cuff tendinopathy. These include tendon compression from extrinsic and intrinsic causes, tendon underuse and overuse, ${ }^{5-11}$ genetics, ${ }^{12}$ evolutionary adaptations ${ }^{13}$ and nutrition. ${ }^{1415}$ To date, a definitive understanding of the pathoaetiology of rotator cuff tendinopathy has not been possible because of equivocal and insufficient research evidence. A review of anatomy, function and pathology of the rotator cuff has recently been published. ${ }^{16}$ Cook and Purdam ${ }^{17}$ recently presented a generic model to define the continuum of tendon pathology. This new paradigm involves staging pathology chronologically within a clinical, histological and imaging framework. The continuum involves a transition from normal tendon to currently irreversible tendon pathology. Variations of the generic model may be required because of regional anatomical and/ or biomechanical considerations. The purpose of this review is to discuss the relevance of this new generic model with regard to rotator cuff tendinopathy and to propose management options for each of the stages described by Cook and Purdam. ${ }^{17}$

\section{ROTATOR CUFF TENDINOPATHY: A NEW MODEL}

The rotator cuff tendinopathy model (detailed in fig 1) is based on the generic model presented by Cook and Purdam ${ }^{17}$ and involves placing normal rotator cuff tendon as the optimal functional unit. This is defined as a structurally sound tendon that is pain-free and capable of performing the normal functional tasks required by the individual. Within this model, the tendon unit has the capacity to positively adapt to appropriate and graduated stress.

Underloaded tendon occurs when the rotator cuff does not receive appropriate physiological stress. Although this may occur throughout the tendon, its presence will be disproportionate and may affect the region of the rotator crescent and the articular side of the supraspinatus tendon. ${ }^{18} 19$ With an appropriate stimulus, an underloaded tendon may return to become a normal tendon. Tendon underuse may result in an imbalance of matrix metalloproteinases (MMPs) and their inhibitors (tissue inhibitors of MMP (TIMPs)), which may eventuate in tendon degradation. Imaging (ultrasound (US) and MRI) investigations may reveal age- and activity-related structural pathology, which in this stage of the continuum may remain asymptomatic. The normal rotator cuff tendon and the underloaded tendon, if subject to an activity level in excess of that normally placed on the tendon, may subject the tissue to overload. If the overload is transient, which may be identified on MRI as in an increase in tendon volume due to increased bound water content, the tendon may return to its preloaded state. This state termed normal tendon overload is painfree and is a normal response to loading rotator cuff tissue through activity and exercise.

However, the increased volume under the coracoacromial arch may potentially increase the strain in the coracoacromial ligament. Because of its trapezoidal shape and the relatively smaller surface area on the acromial side, strain within the ligament may potentially lead to the development of acromial traction spurs. The development of these osteophytes may depend on load and the anatomy of the region. ${ }^{20}$ Continuous loading in this state may have an either positive or negative effect on the tendon. This will depend on 


\section{Rotator cuff tendinopathy: a model for the continuum of pathology}

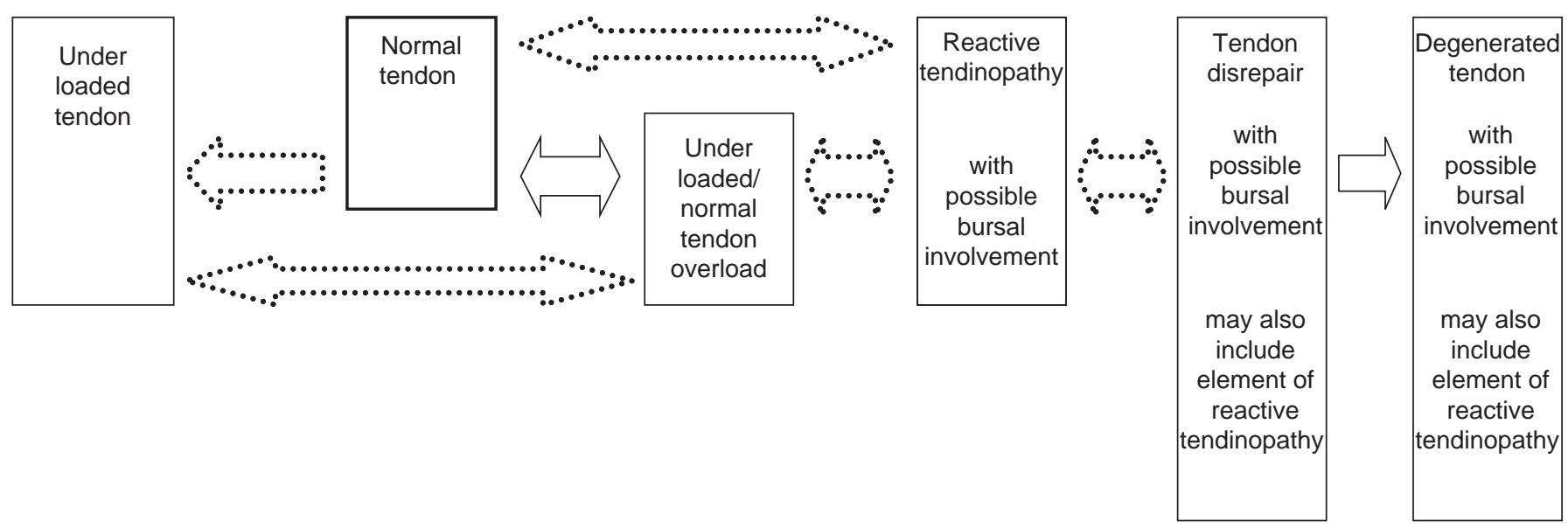

Figure 1 Rotator cuff tendinopathy: a model for the continuum of pathology. Dotted arrows, potentially reversible; solid two-directional arrows, reversible; solid single-directional arrows, irreversible; dotted single-directional arrows, irreversible without going through an intermediate step.

the intensity, volume and frequency of the load applied to the tendon. If applied in a graduated and controlled manner, the loading will lead to a tendon capable of withstanding greater stress.

If the applied load exceeds the physiological capacity of the rotator cuff, the effect may be tendon upregulation. The first stage may be reactive tendinopathy and is predominantly seen in an acutely overloaded tendon. This stage may involve the SAB tissue, where abnormal neuropeptide (substance P) and cytokine levels have been reported. ${ }^{21} 22$

Although there may be no substantial areas of tendon degeneration, there will be increased swelling within the tendon, and it possibly involves bursal effusion. Cook and Purdam ${ }^{17}$ have suggested that this stage occurs following a burst of unaccustomed activity and exists as a transition phase between normal tendon and tendon disrepair. This stage is probably driven by an increased activation of tenocytes, whose first response is driving a deposition of proteoglycans with larger molecular weight such as aggrecan.

Pain may be present, and this may be constant or intermittent and probably position and activity dependent. Constant pain and/or night pain may further implicate the $S A B .^{23}$ In this stage, failure of the rotator cuff to control superior translation of the humeral head may lead to a secondary irritation of the superior fibres of the tendon against the coracoacromial ligament and the undersurface of the acromion. ${ }^{24}$ Because of the upregulation of the vascular endothelial growth factor in the early stages of rotator cuff tendon overuse, ${ }^{16}$ neovascularity may be present.

Cook and Purdam ${ }^{17}$ defined the next stage in the continuum as tendon disrepair. This will involve essentially the same spectrum of clinical symptoms as reactive tendinopathy. The symptoms may be more commonly associated with movement and activity. Tendon disrepair may be characterised by substantial areas of swelling, tendon degeneration, hypoechoic areas on greyscale US imaging that correspond with disorganisation of the matrix, increases in ground substance and some separation of the collagen fibres. It will also be observed as swelling and increased signal on MRI. A bursal reaction characterised by effusion and areas of neovascularity within the tendon and bursa may be present. The presence of neovascularity may be more evident if examined with the tendon off stretch, following an activity or after heating.

Cook and Purdam ${ }^{17}$ have classified the final stage as degenerated tendon, and for the rotator cuff, this will be associated with substantial structural failure in the form of large partial-thickness, full-thickness and massive rotator cuff tears. ${ }^{25}$ In the late stages, there may also be radiological evidence of glenohumeral and acromial degenerative changes.

\section{STAGING TREATMENT WITHIN THIS MODEL}

Patient education, pain reduction, tendon load management (unloading and reloading) and re-injury prevention form the basis of symptomatic rotator cuff tendon rehabilitation. In addition to this, consideration should be given to the possibility of SAB involvement associated with the presenting symptoms. To maintain optimal health and function, tendons require appropriate ongoing mechanical stimulation. Chronically underloaded tendon in a sedentary population may result in asymptomatic degeneration and tears, which will increase with age. This may occur as a result of alterations in the concentrations of the MMPs and TIMPs. It is not currently understood why elite-level athletes develop asymptomatic rotator cuff pathology. ${ }^{26}$ It may be due to overuse or fluctuating periods of activity and relative rest influencing MMP and TIMP concentrations. It may also be due to the relatively low innervation in tendons that do not produce a pain response in the presence of structural pathology until a specific threshold is reached. Treatment for an underloaded rotator cuff involves a tendon-reloading programme in a controlled and graduated manner. The management for a structurally and functionally normal rotator cuff involves maintenance of physical activity with appropriate controlled and graduated increase in tendon loading as required. To maximise the benefit of tendon-loading 


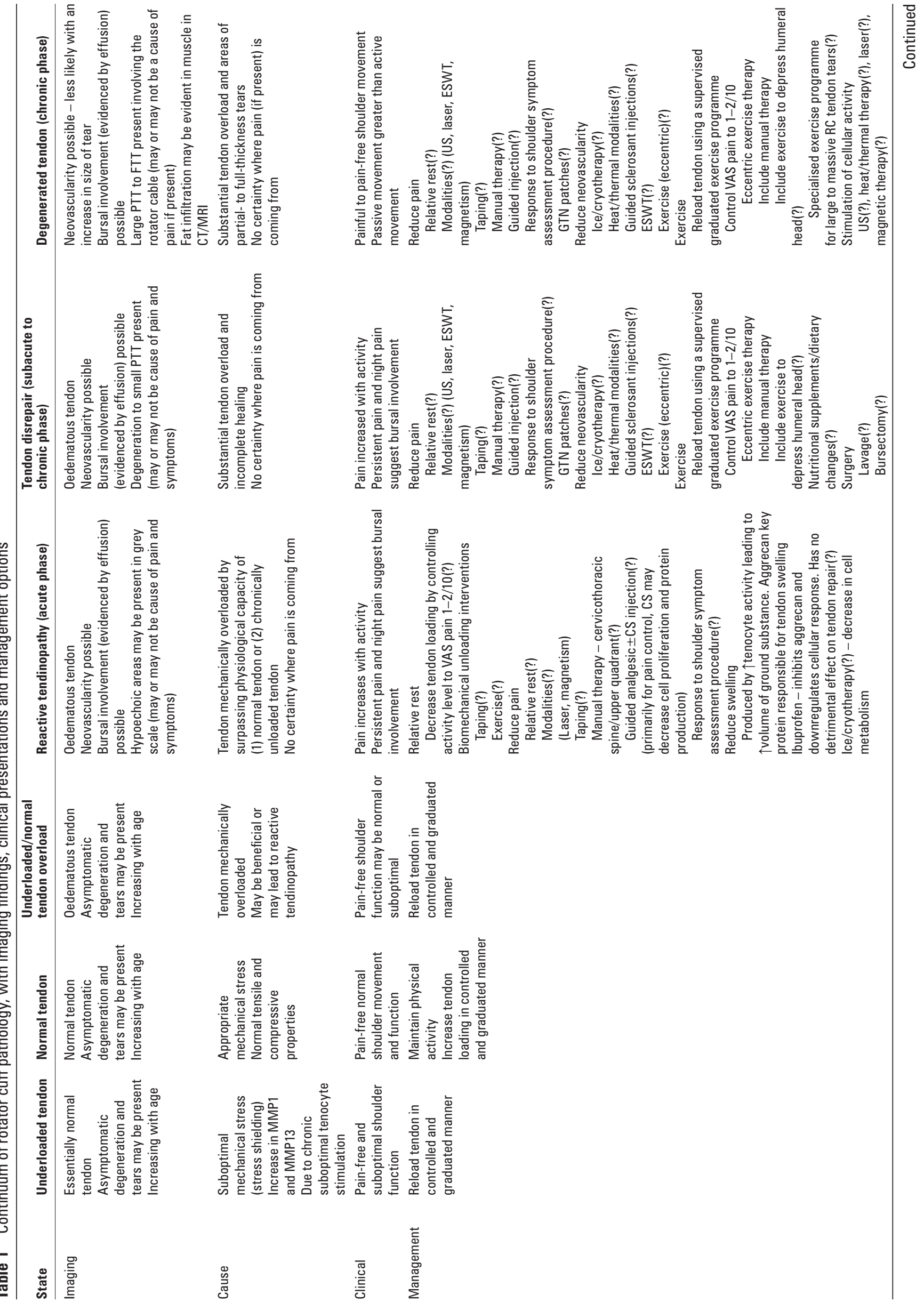




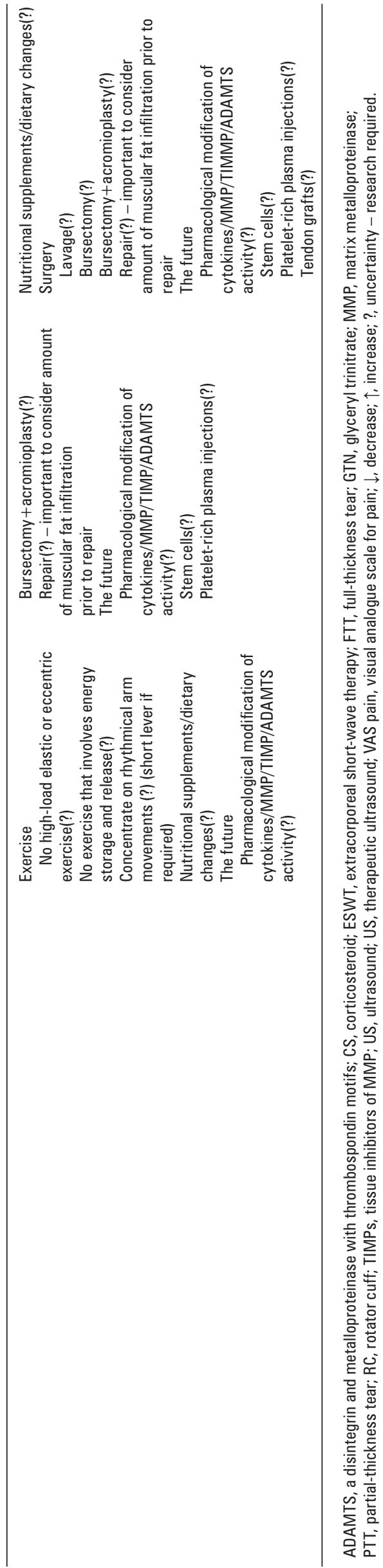

activities, the load, duration and intensity of activity placed on the rotator cuff tendons must be carefully controlled, structured and monitored.

Load reduction, together with pain management, is a key component in the management of reactive tendinopathy. Evidence-based guidance is not currently available. One suggestion requiring vigorous scientific enquiry would be to reduce activity level to control pain to a level of 1 to 2 out of 10 (worst imaginable pain) on a visual analogue scale of pain. Additionally, during this period of relative rest, modalities, taping and manual therapy may be considered to reduce pain and restore shoulder movement and function. The inclusion of these procedures, at this stage of management, is not substantiated by definitive research evidence, and ongoing scientific enquiry is essential. Other methods of assessing the shoulder in order to determine possible management options, including pain reduction and restoration of movement, have been suggested. ${ }^{27}$ The presence of intratendinous swelling is a potential feature of this stage of tendon pathology. Selective non-steroidal anti-inflammatory drugs appear to inhibit tendon cell proliferation and matrix glycosaminoglycan synthesis, ${ }^{28}$ and the synthesis of aggrecan may be inhibited by the non-steroidal anti-inflammatory drug, ibuprofen. ${ }^{29}$ Guided SAB injections may help reduce pain and improve function. ${ }^{30}$ Uncertainty exists as to whether injections should include analgesic or analgesic and steroid. ${ }^{31-33}$ Although yet to be substantiated, cryotherapy may also reduce swelling. Exercises to reduce superior migration of the humeral head on the glenoid fossa may be beneficial. High-load eccentric exercises and fast concentric/eccentric regimes should probably be avoided. Dietary changes may be beneficial to improve tendon health and symptoms. ${ }^{14} 15$

Ongoing pain management and the other components of management for reactive tendinopathy remain important components for the treatment of tendon disrepair. Graduated tendon reloading including concentric, isometric and eccentric exercise is introduced during this stage. Evidence exists that glenohumeral external and internal rotations have opposing effects on subacromial pressure ${ }^{34}$ with external rotation lowering pressure. Additionally, exercises to depress the humeral head may also be warranted. ${ }^{27}$ Failure of an exercise programme to achieve the desired outcome may necessitate other management options such as glyceryl trinitrate patches, ${ }^{35}$ injection therapy and possibly surgery. Conservative treatment should generally be considered before surgical intervention. ${ }^{36} 37$ All interventions require vigorous scientific investigation. Evidence exists from other regions that reducing the expression of neovascularity is associated with a reduction in tendon symptoms. ${ }^{38}$ A number of therapeutic techniques such as cyrotherapy, thermal modalities and exercise may contribute to reducing neovascularity. The relationship between neovascularity and symptoms arising from rotator cuff tendon pathology and the SAB is yet to be established, and if a definitive correlation exists, then research designed to investigate the best methods of reducing the expression of neovascularity in a timely manner without resulting in short- or long-term tendon damage is required.

Pain control and restoring normal movement are the principal aims of managing degenerated tendon. ${ }^{39}$ Clinical investigations have suggested that even in the presence of substantial structural pathology, range of movement and power may be improved when pain is reduced ${ }^{40-42}$ and management options described for tendon disrepair are relevant for tendon degeneration. Another key factor that may positively influence outcome is the presence of a functioning rotator cable. ${ }^{19}$ The decision to

政

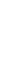

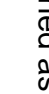


attempt a surgical repair of the tear should be informed on an assessment of the (1) individual patient's functional requirements, (2) size of the tear and (3) the amount of fat infiltration into the muscle because the presence of fatty streaks has been associated with negative surgical outcomes. ${ }^{43} 44$

Future advances in pharmacology, stem cell research and tendon grafts may lead to advances in the management of degenerated tendons and other aspects of the continuum of rotator cuff pathology. Table 1 summarises the continuum of rotator cuff pathology, with possible imaging findings, clinical presentations and management options.

\section{CONCLUSION}

Recently, a new and generic model detailing the continuum of tendon pathology has been proposed. This model is relevant for the rotator cuff and provides a framework to stage the continuity of rotator cuff disease. Furthermore, it provides a structure to identify the substantial deficiencies in our knowledge base and areas, where research would improve our understanding of the pathological and repair process, together with assessment and management. Cook and Purdam ${ }^{17}$ have suggested that the strength of their generic model will be tested in its ability to incorporate and adapt to emerging research. This also applies for the rotator cuff tendinopathy continuum model presented in this paper.

Competing interests None.

Provenance and peer review Not commissioned; externally peer reviewed.

\section{What is already known on this topic}

- Rotator cuff tendinopathy is common and associated with substantial morbidity. Conflicting theories pertaining to the pathogenesis of rotator cuff tendinopathy and bursal pathology exist.

\section{What this study adds}

- The review is presented attempting to describe a model for the continuum of rotator cuff tendinopathy. The continuum involves a transition from normal tendon to currently irreversible tendon pathology. The stages involve asymptomatic tendon, normal tendon overload, reactive tendinopathy, tendon disrepair and degenerative tendinopathy. The model describes a corresponding aetiological, clinical, histological and imaging framework for the various stages, together with a framework for management.

\section{REFERENCES}

1. Richardson AB, Jobe FW, Collins HR. The shoulder in competitive swimming. Am J Sports Med 1980;8:159-63.

2. Ciullo JV, Stevens GG. The prevention and treatment of injuries to the shoulder in swimming. Sports Med 1989;7:182-204.

3. Feltner $\mathbf{M}$, Dapena J. Three-dimensional interactions in a two-segment kinetic chain. Part I: General model. Int J Sport Biomech 1989:5:403-19.
4. Dillman CJ, Fleisig GS, Andrews JR. Biomechanics of pitching with emphasis upon shoulder kinematics. J Orthop Sports Phys Ther 1993;18:402-8.

5. Vogel KG. Tendon structure and response to changing mechanical load. J Musculoskelet Neuronal Interact 2003;3:323-5; discussion 333-4.

6. Bunata RE, Brown DS, Capelo R. Anatomic factors related to the cause of tennis elbow. J Bone Joint Surg Am 2007;89:1955-63.

7. Lyman J, Weinhold PS, Almekinders LC. Strain behavior of the distal achilles tendon: implications for insertional achilles tendinopathy. Am J Sports Med 2004;32:457-61

8. Thornton GM, Shao X, Chung M, et al. Changes in mechanical loading lead to tendon-specific alterations in MMP and TIMP expression: influence of stressdeprivation and intermittent cyclic hydrostatic compression on rat supraspinatus and Achilles tendons. Br J Sports Med 2008; In Press.

9. Soslowsky LJ, Thomopoulos S, Esmail A, et al. Rotator cuff tendinosis in an animal model: role of extrinsic and overuse factors. Ann Biomed Eng 2002:30:1057-63.

10. Perry SM, Mcllhenny SE, Hoffman MC, et al. Inflammatory and angiogenic mRNA levels are altered in a supraspinatus tendon overuse animal model. J Shoulder Elbow Surg 2005;14:79S-83S.

11. Riley GP, Curry V, DeGroot J, et al. Matrix metalloproteinase activities and their relationship with collagen remodelling in tendon pathology. Matrix Biol 2002;21:185-95

12. Harvie $\mathbf{P}, 0$ stlere SJ, Teh J, et al. Genetic influences in the aetiology of tears of the rotator cuff. Sibling risk of a full-thickness tear. J Bone Joint Surg Br 2004;86:696-700.

13. Lewis $\mathbf{J}$, Green A, Yizhat Z, et al. Subacromial impingement syndrome: has evolution failed us? Physiotherapy 2001;87:191-8.

14. Radák Z, Takahashi R, Kumiyama A, et al. Effect of aging and late onset dietary restriction on antioxidant enzymes and proteasome activities, and protein carbonylation of rat skeletal muscle and tendon. Exp Gerontol 2002;37:1423-30

15. Lewis JS, Sandford FM. Rotator cuff tendinopathy: is there a role for polyunsaturated fatty acids and antioxidants? J Hand Ther 2009;22:49-55; quiz 56 .

16. Lewis JS. Rotator cuff tendinopathy. Br J Sports Med 2009;43 236-41.

17. Cook JL, Purdam CR. Is tendon pathology a continuum? A pathology model to explain the clinical presentation of load-induced tendinopathy. Br J Sports Med 2009:43:409-16.

18. Bey MJ, Song HK, Wehrli FW, et al. Intratendinous strain fields of the intact supraspinatus tendon: the effect of glenohumeral joint position and tendon region. J Orthop Res 2002;20:869-74.

19. Burkhart SS, Esch JC, Jolson RS. The rotator crescent and rotator cable: an anatomic description of the shoulder's "suspension bridge". Arthroscopy 1993:9:611-16.

20. Edelson JG, Taitz C. Anatomy of the coraco-acromial arch. Relation to degeneration of the acromion. J Bone Joint Surg Br 1992;74:589-94.

21. Gotoh M, Hamada K, Yamakawa H, et al. Increased substance P in subacromial bursa and shoulder pain in rotator cuff diseases. $J$ Orthop Res 1998;16:618-21.

22. Sakai H, Fujita K, Sakai Y, et al. Immunolocalization of cytokines and growth factors in subacromial bursa of rotator cuff tear patients. Kobe J Med Sci 2001;47:25-34.

23. Santavirta S, Konttinen YT, Antti-Poika I, et al. Inflammation of the subacromial bursa in chronic shoulder pain. Arch Orthop Trauma Surg 1992;111:336-40.

24. Lazarus MD, Yung SW, Sidles JA, et al. Anterosuperior humeral head displacement: limitation by the coracoacromial arch. J Shoulder Elbow Surg 1996:5:S7.

25. Jozsa L, Kannus P. Human tendons: anatomy, physiology and pathology. Champaign, Illinois, USA: Human Kinetics, 1997.

26.. Miniaci A, Mascia AT, Salonen DC, et al. Magnetic resonance imaging of the shoulder in asymptomatic professional baseball pitchers. Am J Sports Med 2002;30:66-73.

27. Lewis JS. Rotator cuff tendinopathy/subacromial impingement syndrome: is it time for a new method of assessment? Br J Sports Med 2009;43:259-64.

28. Riley GP, Cox M, Harrall RL, et al. Inhibition of tendon cell proliferation and matrix glycosaminoglycan synthesis by non-steroidal anti-inflammatory drugs in vitro. $J$ Hand Surg Br 2001;26:224-8.

29. Tsai WC, Tang FT, Hsu CC, et al. Ibuprofen inhibition of tendon cell proliferation and upregulation of the cyclin kinase inhibitor p21CIP1. J Orthop Res 2004:22:586-91

30. Henkus HE, Cobben LP, Coerkamp EG, et al. The accuracy of subacromial injections: a prospective randomized magnetic resonance imaging study. Arthroscopy 2006;22:277-82

31. Plafki C, Steffen R, Willburger RE, et al. Local anaesthetic injection with and without corticosteroids for subacromial impingement syndrome. Int Orthop 2000:24:40-2. 
32. Alvarez CM, Litchfield R, Jackowski D, et al. A prospective, double-blind, randomized clinical trial comparing subacromial injection of betamethasone and xylocaine to xylocaine alone in chronic rotator cuff tendinosis. Am J Sports Med 2005;33:255-62.

33. Ekeberg OM, Bautz-Holter E, Tveitå EK, et al. Subacromial ultrasound guided or systemic steroid injection for rotator cuff disease: randomised double blind study. BMJ 2009;338:a3112.

34. Werner CM, Blumenthal S, Curt A, et al. Subacromial pressures in vivo and effects of selective experimental suprascapular nerve block. J Shoulder Elbow Surg 2006;15:319-23.

35. Murrell GA. Using nitric oxide to treat tendinopathy. Br J Sports Med 2007:41:227-31.

36. Haahr JP, Østergaard S, Dalsgaard J, et al. Exercises versus arthroscopic decompression in patients with subacromial impingement: a randomised, controlled study in 90 cases with a one year follow up. Ann Rheum Dis 2005:64:760-4.

37.. Haahr JP, Andersen JH. Exercises may be as efficient as subacromial decompression in patients with subacromial stage II impingement: 4-8years' follow-up in a prospective, randomized study. Scand J Rheumatol 2006;35:224-8.
38. Alfredson $\mathbf{H}$, Ohberg L. Sclerosing injections to areas of neo-vascularisation reduce pain in chronic Achilles tendinopathy: a double-blind randomised controlled trial. Knee Surg Sports Traumatol Arthrosc 2005;13:338-44.

39. Ainsworth R, Lewis JS. Exercise therapy for the conservative management of full thickness tears of the rotator cuff: a systematic review. Br J Sports Med 2007:41:200-10.

40. Ben-Yishay A, Zuckerman JD, Gallagher M, et al. Pain inhibition of shoulder strength in patients with impingement syndrome. Orthopedics 1994;17:685-8.

41. Brox JI, Røe C, Saugen E, et al. Isometric abduction muscle activation in patients with rotator tendinosis of the shoulder. Arch Phys Med Rehabil 1997;78:1260-7.

42. Steenbrink F, de Groot JH, Veeger HE, et al. Pathological muscle activation patterns in patients with massive rotator cuff tears, with and without subacromial anaesthetics. Man Ther 2006:11:231-7.

43. Goutallier D, Postel JM, Gleyze P, et al. Influence of cuff muscle fatty degeneration on anatomic and functional outcomes after simple suture of fullthickness tears. J Shoulder Elbow Surg 2003;12:550-4.

44. Liem D, Lichtenberg S, Magosch P, et al. Magnetic resonance imaging of arthroscopic supraspinatus tendon repair. J Bone Joint Surg Am 2007;89:1770-6 